\title{
Incidencia de las Consejerías Educativas en el instituto "Rubén Darío", Nueva Guinea, 2017
}

\author{
Educational Counseling Incidence at Rubén Darío Institute, Nueva Guinea, 2017
}

\author{
Juan de Dios Castellón Ramos ${ }^{1}$ \\ Jessenia del Carmen Guzmán Chévez ${ }^{2}$ \\ Consuelo Lizeth Blandón Jirón ${ }^{3}$
}

\section{Resumen}

Esta investigación tuvo como propósito analizar el impacto de las Consejerías de las Comunidades Educativas en el proceso de enseñanza aprendizaje, Instituto "Rubén Darío", Nueva Guinea. El estudio se desarrolló desde un enfoque cualitativo, se hizo uso de técnicas propias de este enfoque como entrevistas, grupos focales y observaciones. Según el nivel de profundidad es descriptiva, porque se describe la incidencia del trabajo que realizan las consejerías de las comunidades educativas. Según el alcance temporal es de tipo transversal porque aborda únicamente el 2017.

Al finalizar la investigación se concluye que, los padres y madres de familia han asumido mayores compromisos con la educación de sus hijos, los estudiantes han mejorado su disciplina, las charlas educativas han sido un factor fundamental para fortalecer los procesos educativos, se ha contado con el apoyo de instituciones del Estado y organizaciones para brindar charlas educativas. También se encontraron aspectos negativos como poca asistencia de padres y madres de familia en las charlas y reuniones.

Palabras clave: Impacto; consejerías; estrategias; incidencia; comunidades educativas; enseñanza aprendizaje; efectividad.

\section{Abstract}

The purpose of this research was to analyze the impact of the Educational Counseling Communities in the teaching-learning process at "Rubén Darío" Institute, Nueva Guinea.

The study was developed from a qualitative approach, using techniques of this approach such as interviews, focus groups and observations. According to the depth level, it is descriptive, because it describes the incidence of the work carried out by the educational counseling communities. According to the temporal scope, it is cross-sectional because it only addresses 2017 school year.

At the end of the research, it is concluded that parents have made greater commitments to the education of their children, students have improved their discipline, educational talks have been a fundamental factor in strengthening the teaching-learning processes, and it has been counted on the support of State institutions and organizations to provide the educational talks. Negative aspects were also found, such as poor attendance by fathers and mothers in the talks and meetings.

Keywords: Impact; counseling; strategies; incidence; educational communities; teaching learning; effectiveness.

\footnotetext{
1 Máster en Docencia Universitaria. Docente Horario URACCAN, Recinto Nueva Guinea. Correo: jcastellonr@gmail.com ORCID: https://orcid.org/0000-0001-53673557

2 Máster en Docencia Universitaria. Docente Educación Secundaria.

3 Máster en Docencia Universitaria. Secretaria Académica, URACCAN, Recinto Nueva Guinea. Correo: consuelouraccan@gmail.com ORCID: https://orcid.org/0000-0003-0301-489X
}

Recibido: 25/10/2018- Aprobado: 28/05/2020 


\section{Introducción}

La educación tiene que ver con la adquisición de un conjunto de elementos fundamentales para la formación profesional tales como: conocimientos técnicos, científicos, capacidades, valores y actitudes que brinden las herramientas necesarias para enfrentarse en su mundo laboral con calidad y eficiencia.

La educación en Nicaragua se da en tres niveles: Educación primaria, educación media o secundaria y educación superior o universitaria. La educación secundaria es una etapa de transición para ingresar a la educación superior (Universidad), donde el estudiante debe adquirir conocimientos, habilidades y valores para enfrentar una nueva experiencia, es aquí donde las consejerías de las Comunidades Educativas desempeñan una labor importante en la preparación de estos jóvenes y adolescentes para enfrentar un nuevo reto.

El Instituto Nacional de Participación Educativa «Rubén Darío» (INPERD), ubicado en el área urbana de la ciudad Nueva Guinea RACCS, imparte educación secundaria en las modalidades: Matutino, Vespertino y Sabatino y de este centro egresan una gran cantidad de bachilleres a las diferentes universidades del municipio y del país.

Es esta la razón de la investigación "Incidencia de las Consejerías de las comunidades Educativas en el proceso educativo en el Instituto "Rubén Darío", Nueva Guinea, 2017", la que tiene como propósito verificar la efectividad que ha tenido esta forma de dirigir la educación en este centro educativo y cuales han sido los logros obtenidos.

Consideramos que los resultados encontrados serán de gran utilidad para la dirección y docentes del Instituto "Rubén Darío" porque servirá como un documento de referencia para evaluar el trabajo realizado por las Consejerías de las Comunidades Educativas y mejorar las debilidades y seguir fortaleciendo los aspectos positivos, a los padres y madres de familia y toda la comunidad educativa como un elemento de motivación para involucrarse más en los procesos educativos del estudiantado.

\section{Revisión de la literatura}

\section{Consejerías de las Comunidades Educativas}

Según el Ministerio de Educación (MINED), en el documento: Estrategias para la organización y el funcionamiento de las Consejerías de las Comunidades Educativas (2016), las define como:

Un componente de las estrategias de resguardo de la tranquilidad de las familias que funcionan en los centros educativos a partir del protagonismo de maestros y maestras, estudiantes, madres, padres de familia con los principios y valores de nuestro gobierno (p.4).

Esta estrategia pretende involucrar a diferentes actores sociales en la educación del estudiantado, donde todos puedan velar por la práctica de valores que contribuyan a formar una sociedad con buenos principios y comportamiento, es interesante que haya integración de todas las partes que conforman la comunidad educativa para lograr los objetivos del gobierno.

\section{Funciones, responsabilidades y líneas de trabajo de las Consejerías de las Comunidades Educativas}

El manual de estrategias para la organización y el funcionamiento de las Consejerías de las Comunidades Educativas (2016, p.9), expresa que "las comisiones están distribuidas por centro, por modalidad y por aula, cada una desempeña funciones específicas". 


\section{Comisión representativa de cada centro:}

1. Impulsar las actividades de promoción de valores, prevención, capacitación, detección, atención primaria, acompañamiento y derivación de situaciones que enfrenta la comunidad educativa.

2. Planificar, organizar, coordinar, convocar, desarrollar, acompañar, dar seguimiento y evaluar actividades con docentes, estudiantes, padres, madres y tutores.

3. Llevar registro de las acciones planificadas y desarrolladas por las Consejerías de las Comunidades Educativas (CCE), de su centro en el libro de acta.

4. La o el director o subdirector del centro será el responsable de la comisión y llevará informe mensual a la Delegación municipal.

5. Esta comisión recibirá los diferentes casos que sean remitidos por las diferentes comisiones por modalidad en cada turno.

6. Referirán y acompañarán a los estudiantes, o familia a la atención especializada en casos que se refiera, estableciendo las coordinaciones correspondientes a nivel local.

7. Se deberán reunir una vez al mes para planificar y dar seguimiento al desarrollo de las acciones.

8. Brindan logros y avances de las acciones de las Consejerías de las Comunidades Educativas, en los matutinos, en los encuentros con padres, madres y tutores y en los encuentros para entrega de notas en las cortes evaluativas.

\section{Comisiones por modalidad}

De acuerdo a las Estrategias para la Organización y el funcionamiento de las CCE (2016, p.10), establece una serie de responsabilidades que deben cumplir las comisiones:

1. Impulsar las actividades de promoción de valores, prevención, capacitación, detección, atención primaria, acompañamiento y derivación de situaciones que enfrenta la comunidad educativa en su turno y modalidad.

2. Recibir la información que suministren la comisión por aula.

3. Llevar un registro de las acciones que se planifican y desarrollan en su respectiva modalidad y turno.

4. Remitir los casos o situaciones que ameriten atención, especializada a la comisión representativa del centro educativo.

5. Informar mensualmente a la comisión representativa de la ejecución de las acciones desarrolladas.

6. Los padres, madres o tutores que forman parte de las comisiones por modalidad serán convocados en el centro educativo cuando las situaciones lo ameriten.

7. Se deberán reunir una vez al mes para planificar, identificar, desarrollar, dar seguimiento y evaluar el desarrollo de las acciones. 


\section{Consejeras y consejeros por aula}

Según El Ministerio de Educación, (MINED; 2016, p.11), mediante Manual de Estrategias para la Organización y el Funcionamiento de las Consejerías de las Comunidades Educativas, establece que las consejeras y consejeros por aula:

1. Serán los enlaces entre las distintas aulas, y la comisión por modalidad o representativa, debiendo informar a las distintas comisiones acerca de las situaciones que sucedan en las aulas.

2. Serán quienes desarrollen o garanticen las sesiones de reflexión con estudiantes los días miércoles de cada semana por aula, así como los encuentros por padres y madres de familia o tutores o tutoras una vez al mes.

De acuerdo al manual de las estrategias para la organización y el funcionamiento de las Consejerías de las Comunidades Educativas (2016, p.12), estas tienen la responsabilidad de apoyar y dar seguimiento a las siguientes actividades:

1. Capacitaciones en temas de promoción y práctica de valores con la comunidad educativa.

2. Encuentros con madres, padres de familia, tutores y tutoras, abordándose una temática el tercer viernes de cada mes.

3. Sesiones entre estudiantes con sus docentes de aulas y guías para promoción y práctica de valores.

4. Encuentros de capacitación con los docentes una vez al mes.

5. Organización y desarrollo de actividades deportivas y culturales.

6. Programación y realización de actividades preventivas para evitar situaciones difíciles.

7. Detección, atención y acompañamiento a niñas, niños, adolescentes y jóvenes que sufran situaciones que afecten su desempeño escolar.

8. Promoción de una cultura de armonía, solidaridad y tranquilidad, que contribuyan a vivir sanos y saludables en los centros educativos.

9. Congresos de las consejerías de las Comunidades Educativas a nivel municipal, departamental y nacional.

Interés de los padres, madres de familia, estudiantes y docentes en la integración de las consejerías de las comunidades educativas

La educación debe ser un tema de interés para padres y madres de familia como principales formadores de valores en el hogar, los estudiantes como protagonistas reciben la educación, los docentes y dirección quienes dirigen el proceso de enseñanza, al igual que las demás autoridades que diseñan las políticas y estrategias educativas, es un trabajo en conjunto donde debe unir esfuerzos demostrando interés y voluntad para cumplir con esta intención de superar obstáculos en los procesos educativos.

$\mathrm{Al}$ respecto Leiva et al (2010), plantean que:

El o la Consejera necesita ser una persona abierta al conocimiento y dispuesta a prepararse en diferentes temas y técnicas para facilitar los procesos de reflexión sobre los problemas y la toma de decisión de los cambios que las personas de forma voluntaria quieren hacer en sus vidas. El 
consejero o consejera debe articular acciones con los actores de la comunidad educativa (director/a, docentes, padres y madres de familia o tutores y estudiantes), a fin de que se comprometan con la Consejería Escolar y no la consideren como una tarea exclusiva del docente (p.71).

Este planteamiento da a entender que las personas que integran las consejerías deben poseer ciertas cualidades y sobre todo disponibilidad para enfrentar esta tarea y dar respuestas a diferentes situaciones que se presenten en los centros educativos, para estos recibe charlas, talleres, foros, entre otras actividades para obtener nuevos conocimientos y adquirir compromisos para cumplir con sus funciones encomendadas.

\section{Rol del director o directora en la escuela}

Hablando en términos administrativos el director o directora es la máxima autoridad en el centro de educación, por lo tanto, su nivel de responsabilidad incide mucho en el proceso educativos en el estudiantado.

Spence (1992), manifiesta que:

En la cima de la jerarquía de funciones está el Director, el” jefe”, de la escuela. Sus deberes incluyen el manejo general de la escuela y su programa de instrucción, el trato con estudiantes y maestros y el mantenimiento de relaciones con grupos sociales y sistemas fuera de la escuela, tanto profesionales (sus superiores en la oficina central), como no profesionales (padres y grupos de interés de la sociedad (p.181)

En la actualidad, el director o directora sigue siendo el máximo representante de la escuela, quien dirige el funcionamiento de todo el centro educativo, con el nuevo modelo de responsabilidad compartida, como su nombre lo dice, estas funciones y tareas deben ser resueltas en conjunto con los demás miembros que conforman la comunidad educativa, donde cada uno de ellos tiene un grado de compromiso en este proceso.

\section{Rol de los docentes en los procesos educativos}

Los docentes desempeñan una gran labor en el proceso educativos, son quienes interactúan de manera directa a la hora de impartir los contenidos y crean esos espacios de socialización entre los estudiantes.

García \& Chavarría (2010; p. 23), plantean que: La planificación del docente se ubica en el nivel más minucioso y preciso de planificación educativa, la planificación educativa es una práctica económica y política en el desarrollo de la ideología en la teoría económica de la educación. La educación es el motor que impulsa el desarrollo, sin educación no hay desarrollo.

\section{Rol de los padres y madres de familia en los procesos educativos de los estudiantes}

García y Chavarría (2010), plantean que:

La familia cumple con el cometido de mantener y socializar al niño o niña, al mismo tiempo que contribuye a mantener el control social, constituyéndose en el primer agente de socialización, y no solo por ser el primero en actuar sino, fundamentalmente, por el carácter cualitativo de su influencia (p.28).

Muchas madres y padres de familia no cumplen con su función en la parte que le corresponde en el proceso educativos de sus hijos e hijas en la escuela como: revisar y ayudar realizar las tareas, asistir a las reuniones, estar pendiente del comportamiento de sus hijos, participar en actividades extraescolares, en 
jornadas de limpieza, elaboración de huertos escolares, velar por el cuido y mantenimiento del centro, entre acciones que ayuden al desarrollo socioeducativo.

\section{Rol de los estudiantes en el proceso educativo}

Los estudiantes se consideran el núcleo fundamental en la escuela, porque son quienes reciben la enseñanza y los demás miembros de la comunidad educativa diseñan y ejercen autoridad sobre ellos, Espence (1992), expresa que:

Los estudiantes constituyen el grupo mayoritario en la escuela, están en la parte inferior de la jerarquía de funciones, aunque los estudiantes son los "usuarios de la escuela, y las principales decisiones se adoptan en aras del beneficio de los estudiantes", su opinión cuenta poco en tales decisiones (p.194)

Es importante reconocer que con los nuevos modelos de educación las funciones de los estudiantes no solamente se limitan en cumplir con ciertas tareas como: asistir a clases, cumplir con las actividades asignadas en la escuela, sino que sus opiniones también cuentan prueba de esto es que forma parte de las Consejerías de las Comunidades Educativas como la principal estructura que dirige el proceso de enseñanza.

\section{Estrategias para involucrar a los docentes, estudiantes, padres y madres de familia en las consejerías educativas}

Los padres y madres de familia juegan una labor muy importante en la formación de sus hijos e hijas, al igual que el Ministerio de Educación a través de los docentes, pero ellos deben ser los primeros interesados, pero en muchas ocasiones estos se ausentan de cumplir con esta responsabilidad, es aquí donde se deben implementar estrategias que hagan conciencia para que y se involucren en esta importante tarea,

Quiroz, et. al., (2010, p.73), proponen algunas pautas para orientar a los padres en la educación de sus hijos e hijas.

1. Infórmese más sobre los problemas de aprendizaje. Mientras más se sabe, mas puede ayudarse a sí mismo y a su niño.

2. Participe en reuniones con los consejeros (as) del centro de estudio de su hijo

3. Elogie a su niño cuando le vaya bien

4. Deje que su niño ayude con las tareas domésticas. Estas pueden aumentar su confianza y destrezas concretas.

5. Haga las tareas escolares una prioridad. Lea más cerca de cómo puede ayudar a su niño a tener éxito con las tareas.

6. Ponga atención a la salud mental de su niño y a la suya.

7. Hable con otros padres cuyos niños tienen problemas de aprendizaje, los padres pueden compartir consejos prácticos y apoyo emocional.

8. Reúnase con el personal escolar y ayude a desarrollar un plan educacional para tratar con las necesidades de su niño.

9. Planifique las acciones que su niño necesita. 


\section{Materiales y métodos}

\section{Ubicación del estudio}

Es una investigación de campo que pretende conocer y analizar la incidencia del trabajo que realizan las Consejerías de las Comunidades Educativas en el Instituto Nacional de Participación Educativa "Rubén Darío" (INPERD), ubicado en el área urbana de la ciudad Nueva Guinea, Región Autónoma de la Costa Caribe Sur (RACCS), para esto fue necesario visitar el centro educativo donde se encontraban los sujetos de estudio que brindaron la información necesaria.

\section{Enfoque de la investigación}

El estudio se desarrolló desde un enfoque cualitativo, pues lo que se pretende es indagar y describir la incidencia del trabajo que ejercen las Consejerías de las Comunidades Educativas en el proceso educativos, en el Instituto Nacional de Participación Educativa "Rubén Darío", se utilizaron técnicas que permitieron obtener datos cualitativos y de esta manera cumplir con los objetivos planteados en esta investigación, está sustentado en las siguientes Teorías. Barrantes (1,999), "la investigación cualitativa pone énfasis en la profundidad y sus análisis no necesariamente son traducido a términos matemáticos" (p. 71).

\section{Tipo de investigación}

Se considera descriptiva, porque describe la incidencia del trabajo que realizan las consejerías de las Comunidades educativas en el proceso educativos en el estudiantado del Instituto Nacional de Participación Educativa "Rubén Darío" y se limita a la descripción de los datos sin establecer causas ni relaciones.

\section{Unidad de análisis}

La unidad de análisis está conformada por: Directora del Instituto "Rubén Darío", 4 docentes del turno matutino (2 varones y 2 mujeres), 10 padres y madres de familia, 10 estudiantes del turno matutino, 10 miembros de la Comisión Representativa de Las Consejerías de las Comunidades Educativas, 1 miembra de la Comisión evaluativa del MINED nacional, para un total de 36 informantes.

\section{Criterios de selección}

Para este estudio seleccionaron: Directora del Instituto "Rubén Darío", Responsable de la Comisión Representativa de las Consejerías de las Comunidades Educativas, miembra de la comisión evaluativa del MINED Nacional, docentes de la modalidad regular, de noveno, decimo y undécimo grado, padres y madres de familia con estudiantes en la modalidad regular del turno matutino y que estuvieran presentes en las charlas o reuniones, estudiantes de la modalidad Regular, del turno matutino.

Se excluyen de la selección: A docentes que no fueran de la modalidad regular, del turno matutino y noveno, decimo y undécimo grado, a padres y madres de familia que no asistían a las charlas o reuniones y que no tuviesen hijos en el turno matutino, en noveno, decimo y undécimo grado, a los estudiantes que fueran de la modalidad sabatina o del turno vespertino.

\section{Técnicas e instrumentos}

Se aplicaron a entrevistas a: Directora del Instituto Nacional de Participación Educativa "Rubén Darío" (INPERD) y a cuatro docentes del turno matutino, de Noveno, Décimo y Un Décimo grado, (dos varones y dos mujeres). 
Tres grupos focales: a 10 padres y madres de familia (turno matutino), 10 estudiantes del turno matutino de Noveno, Décimo y Undécimo grado, 10 miembros de las Comisiones de las Comunidades Educativas. Se realizaron 2 observaciones en reunión de padres y madres de familia en el turno matutino.

\section{Análisis y resultados}

\section{Efectividad del trabajo que realizan las Consejerías Educativas}

Los docentes manifestaron que este modelo es bastantes nuevo para hablar de un cambio total, es un proceso que no se logra en poco tiempo, pero se está tratando de cumplir con lo que persigue el programa y que esto debe ser una lucha constante para lograrlo, según una de las docentes entrevistadas, dijo "Pensamos que no ha habido un giro de trecientos sesenta grado porque es formación de valores y tiene que ver con el cambio de actitud de nosotros los maestros, estudiantes, los padres, pero creemos que los estudiantes están mejorando".

Cabe destacar que no todo va a funcionar a la perfección siempre van a darse algunos problemas pero si se ha logrado mucho, tanto docentes y padres de familia reconocen que con esta estrategia y gracias a los esfuerzos hechos en conjunto se pueden notar buenos avances que benefician a la educación como los que se reflejan a continuación:

1. Mayor comunicación entre padres, madres de familia con los docentes y el consejero general. Esto permite conocer el comportamiento tanto en conducta y aprendizaje de sus hijos e hijas.

2. Avances en la disciplina, algunos estudiantes, han mejorado su aspecto y conducta.

3. Los padres están asumiendo más compromisos con relación a la educación de sus hijos.

4. Los padres y madres de familia están más pendiente de las actividades que realizan sus hijos e hijas dentro y fuera del hogar.

5. Se ha mejorado la relación entre padres, madres de familia con sus hijos creando espacios de diálogos para conversar sobre la educación y no solo castigándolos cuando hacen algo malo o indebido.

6. En el caso del estudiantado, se ha mejorado en el uso correcto de la tecnología.

7. Los estudiantes han logrado tomar conciencia de los peligros que se corren al hacer uso inadecuado de la tecnología.

8. Se ha conducido al estudiantado al conocimiento responsable de la sexualidad como condición humana.

9. Se han involucrado en distintas actividades que permiten el fortalecimiento de su adolescencia y la promoción de valores.

10. No se han dado problemas graves de violencia en el centro en los que se tenga que recurrir a las autoridades o a otras instancias, esto significa que se va avanzando en cuanto a la práctica de valores.

11. Los padres y madres de familia están conscientes de la labor que les corresponde a cada uno de ellos en la educación de los estudiantes.

Las charlas educativas han sido un elemento fundamental para alcanzar logros significativos en el proceso educativos, tanto docentes, padres y madres de familia coinciden en que las consejerías son muy importantes porque se abordan temas de interés, para la comunidad educativa, por ejemplo como tratar 
a los hijos, control de los estudiantes en las actividades que realizan dentro y fuera de las aulas de clases, apoyar a los hijos en las actividades y tareas asignadas por los docentes, comunicación en el hogar, autoestima, motivación por el estudio, además hay reuniones de información donde se da a conocer la situación de los estudiantes.

Un docente de los consultados, manifestó que:

Las Consejerías Educativas son muy buenas desde el punto de vista general en donde todos estamos involucrados, tanto el padre de familia el estudiante y los maestros a tratar de buscar cómo mejorar esta situación dado que en algunos casos se han perdido los valores morales, comunitarios, sociales, académicos y de manera general para orientar a los padres de familia y a los estudiantes para que ellos puedan mejorar y tener éxito en un futuro y poder emprender por sí mismos.

Las consejerías educativas buscan involucrar a los padres y madres de familia, en diferentes actividades educativas, promover el rescate de los valores en los estudiantes, motivar para que sean emprendedores y alcanzar éxito en el futuro, de igual manera la Constitución Política de Nicaragua en el Articulo 118, expresa que "El Estado promueve la participación de la familia, de la comunidad y del pueblo en la educación".

\section{Dificultades para el funcionamiento de las Consejerías Educativas}

Es importante destacar los logros sin obviar los problemas que de una y otra manera afectan el trabajo de las Consejerías y por ende la educación en este centro educativo, en entrevistas y conversaciones personales con algunos docentes, se logró identificar algunas dificultades que afectan el buen funcionamiento de este modelo, entre ellos tenemos:

Algunos padres y madres de familia consideran que esto es una pérdida de tiempo porque estos temas son cosas que ya las manejan, un docente comentó que un padre de familia dijo que él ya sabía cómo educar a sus hijos sin necesidad de estas charlas.

\section{Muchos padres de familia no asisten a las charlas educativas y a las reuniones}

Las charlas y reuniones se realizan en días y horas laborales y muchos padres y madres de familia no pueden asistir porque no se les da permiso o le afecta otras actividades que tienen que realizar con relación a su trabajo.

Las charlas se están haciendo cada mes y los padres consideran que es muy corto tiempo, para realizar las consejerías. Los encuentros de padres y madres de familia coinciden en todos los centros y esto impide una mayor participación.

Todo esto permite una escasa participación de padres y madres de familia en las diferentes actividades relacionadas con el quehacer educativo y que viene afectar de manera negativa en la formación de los estudiantes.

\section{Interés de los padres, madres de familia, estudiantes y docentes en la integración de las Consejerías Educativas}

A pesar de algunas limitantes, hay padres y madres muy interesados en la Educación de sus hijos e hijas, así lo manifestó la Responsable de las Consejerías de este centro educativo:

Yo siento que ahora los padres se involucran más porque antes venía te dejaba el chavalo en la secundaria y nunca lo mirabas, pero ahora con los encuentros el padre está más preocupado, por ejemplo, en grupos donde había muchos problemas de disciplina, de rendimiento académico, los 
padres preocupados se ponen al frente y dicen yo voy a llegar ayudar con la disciplina, vienen y se sientan ahí en el aula adentro o afuera y el chavalo se aquieta un poco y trabaja.

Hay diferentes formas de involucrarse en la educación como miembros de las Consejerías de las Comunidades Educativas, tanto docentes, como padres, madres y estudiantes, el presidente de padres de familia del Instituto "Rubén Darío", expresó lo siguiente "Nos involucramos en la medida que participamos en las Asambleas de padres de familia, participamos en las charlas y después compartimos con nuestros hijos en el hogar".

Para el estudiantado, al igual que los padres de familia una forma en que ellos se involucran en el proceso de enseñanza, además de cumplir con sus clases, tareas, entre otras actividades escolares, es compartir conocimientos e información que ellos reciben en las charlas y de esta manera se ayudan unos a otros.

De acuerdo a una estudiante para ella una forma de involucrarse es "Dar información adquirida por nuestros maestros por nuestros y padres, a nuestros compañeros que tal vez no tienen información, entonces nosotros le ayudamos a transmitir información sobre dicha necesidad", otra de las estudiantes, manifestó:

Yo creo que una manera que nos estamos involucrando como estudiantes le decimos a los demás compañeros lo bueno y lo malo que estamos haciendo y así nosotros podemos ir cambiando nuestra forma de pensar y de ver el mundo y así nos estamos ayudando mutuamente en el centro educativo.

Otra manera fundamental de integrarse en este proceso es participar de manera activa en las charlas y reuniones de padres y madres de familia, donde se abordan temas de interés con relación a la educación, en cuanto a la asistencia a estas actividades consideramos que hay bastante debilidad de acuerdo a lo que pudimos observar en vistas al centro y entrevistas aplicadas a los docentes.

En una observación realizada en la sección de Undécimo grado, de 44 padres y madres de familia que conforman este grupo únicamente estuvieron 12 (diez mujeres y dos varones), en otra observación realizada en Décimo grado de 35 padres y madres de familia, asistieron seis mujeres y un varón, como podemos observar en estos datos, quienes más participan son las madres de familia.

Con las entrevistas aplicadas a los docentes también logramos conocer la escasa participación de los padres y madres de familias en las charlas y reuniones, una profesora de décimo grado, manifestó: "En mi caso tengo la cantidad de 35 estudiantes y he llegado a tener en algunas reuniones: 5, 7 y 12 padres de familia".

Hay muchos padres y madres de familia que no se integran en las charlas y reuniones y sus hijos tienen dificultades, así lo manifestó otra de las profesoras entrevistada.

Bueno en las charlas casi no asisten la mayoría de los padres de familia y siempre los padres que vienen son de los hijos que van bien en clases y nosotros deseamos que vengan los padres de los hijos que van mal en las clases, pero siempre los que vienen son los padres que están interesados que los hijos vayan bien.

Esto demuestra que la responsabilidad del padre o madre de familia incide directamente en la educación de sus hijos e hijas porque están más pendientes de las actividades, comportamientos y calificaciones e informados de cómo van en las clases.

\section{Estrategias que contribuyan a la efectividad de la labor de las Consejerías de Educativas en el Instituto "Rubén Darío"}

1. Según los docentes expresaron que las estrategias que se utilizan para involucrar a los padres de familia en el trabajo de las Consejerías de las Comunidades Educativas, son las siguientes: 
2. Cuando no llegan a las charlas, reuniones o entrega de boletines de sus hijas e hijas, se regresa al estudiante el día de clases momentáneamente, mientras regresa el padre, madre de familia o tutor a presentarse o justificarse.

3. Se le envía la invitación previa a la evaluación para que el padre de familia pueda asistir a la reunión que se va a realizar. Esto le permitirá planificarse con anticipación para estar presente.

4. Tenemos un grupo en whatsApp de padres de familia, esto nos permite estar comunicados constantemente con la tecnología.

5. Se les llama por teléfono y se convoca a las reuniones con anticipación.

6. Se les envía razón a los padres y madres de familia con los mismos estudiantes.

7. Mantener buena relación entre docentes con padres y madres de familia.

\section{Conclusiones}

1. El trabajo de las Consejerías de las Comunidades Educativas ha incidido de manera positiva en el proceso educativos en el Instituto "Rubén Darío", aunque no con los resultados esperados porque se considera una estrategia bastante nueva, aunque se pueden visibilizar cambios.

2. Las Comisiones de las Consejerías de las Comunidades Educativas son una herramienta de apoyo para el trabajo del docente.

3. Los padres y madres de familia han mejorado la comunicación con los docentes.

4. Padres y madres de familia han mostrado interés y asumido mayores compromisos con la educación de sus hijos e hijas.

5. Los estudiantes han mejorado su comportamiento y disciplina.

6. Las charlas educativas han sido un factor fundamental para fortalecer el proceso educativos.

7. Se necesitan estrategias que motiven la participación de padres y madres de familia en las charlas y reuniones de sus hijos e hijas.

8. Se ha contado con el apoyo de Instituciones del Estado y organizaciones que han incidido de manera positiva en el proceso educativos en el Instituto "Rubén Darío".

\section{Agradecimiento}

Esta publicación obtuvo el financiamiento de: El Fondo de Asistencia Internacional de los Estudiantes y Académicos Noruegos (SAIH).

\section{Lista de referencias}

Barberena. M, M. M. (2011). El papel del de la Consejería Escolar en el suministro de información objetiva y adecuada para el manejo de una sexualidad sana y responsable en los y las docentes y jóvenes de la Escuela Normal Regional Gregorio Aguilar Barea de la ciudad de Juigalpa . Juigalpa, Chontales, Nicaragua. 
Constitución Política de Nicaragua. (2003). Managua, Nicaragua.

Flores. F, Ponce. L, Palma. M, Pérez. V. (2010). ¿Que Acciones puede Desarrollar el o la Consejera Escolar en la Prevención del Embarazo en las Adolescentes de Educación Secundaria?, IDEUCA. Managua, Nicaragua.

Gadea. M, Palacios, F, Escobar. D \& Briones . J. (2010). Falta de Integración de Padres y Madres de Familia a las Actividades de la Escuela,IDEUCA. Managua, Nicaragua.

GOBIERNO. (2000). CONSTITUCION. MNAGUA.

Gutierrez, M. \&. (2010). Promoción del autoestudio en estudiantes desde la Consejería Escolar,en el Instituto a Distancia Papaturro del Municipio de San Carlos Río, san Juan. Juigalpa, Chontales, Nicaragua.

Hurtado. C, R. O. (2011). Involucramiento de los padres de familia en las tareas educativas de sus hijos e hijas y el rol del docente en el proceso de aprendizaje en el quinto grado de la Escuela Luis Alfonso Velásquez Flores. Juigalpa Chontales, Nicaragua.

IDEUCA, L. M. (2010). Diplomado en Consejería Escolar, Modulo 1, Propedeutico. Managua.

Lopez Avendaño, O. (2007). Sociología de la educación. San José, Costa Rica: EUNED.

Melgosa. (2007). Para adolescentes y padres. Madrid, España: Safeliz.

Navarro, S. G. (2005). El concepto de participación de los padres en el proceso de enseñanza aprendizaje: la perspectiva de agentes compartidos. santiago de Chile.

Quiroz. S, J. G. (2010). Diplomado en Consejería Escolar, Módulo 2, Consejería Psicopedagógica. IDEUCA, 96.

S, R. (2010). Estrategias de Aprendizaje de niños y niñas sin la presencia paterna, 2do grado A, de la Escuela Mixta San Lorenzo. Juigalpa, Nicaragua.

Viñas Cirera, J. (2004). Conflictos en los Centros Educativos. Madrid. 